\title{
Respuesta inicial de la regeneración arbórea luego de la floración y muerte de Chusquea culeou (Poaceae) en bosques andinos del centro-sur de Chile
}

\author{
Early response of tree regeneration after Chusquea culeou (Bambuceae) dieback \\ in Andean old-growth forests of south-central Chile
}

\author{
Ariel A Muñoz a*, Mauro E González a, Constanza Celedón b, Thomas T Veblen c \\ *Autor de correspondencia: ${ }^{a}$ Universidad Austral de Chile, Instituto de Silvicultura, Laboratorio de Ecología de Bosques, \\ Campus Isla Teja, Valdivia, Chile, tel.: 56-63-221228, arielmunoz@live.cl \\ b Universidad de las Islas Baleares, Instituto Mediterráneo de Estudios Avanzados, Islas Baleares, España. \\ ${ }^{c}$ University of Colorado, Department of Geography, Boulder, United States of America.
}

\begin{abstract}
SUMMARY
In temperate rainforests at mid-elevation (> $600 \mathrm{~m}$ a.s.l.) in South-America the Chusquea culeou bamboo (colihue) is the dominant understory species and potentially can impede tree regeneration. Dieback of colihue subsequent to its gregarious flowering may result in an opportunity for tree establishment or growth release of previously suppressed juvenile trees, especially in canopy gaps. In this research we evaluated the bamboo dieback effect on tree species establishment and advance regeneration growth in two old-growth forests. The first forest is dominated by Nothofagus dombeyi-Saxegothaea-conspicua-Dasyphyllum diacantoides and the second is dominated by Araucaria araucana-Nothofagus, located in the Andean Cordillera of south-central Chile. In each forest (San Pablo de Tregua and Quetrupillán) following the gregarious flowering and dieback of colihue, between 2001 and 2003, we recorded new establishment of colihue and tree demographic changes and growth of advance regeneration in gaps with live versus dead colihue. In both forests the mortality of the advance regeneration was higher in gaps with dead colihue. Only in San Pablo was the height growth of Saxegothaea conspicua higher in gaps with dead colihue. New tree establishment did not show differences between flowered and non-flowered conditions. The recruitment of colihue seedlings was higher in gaps with dead colihue in both forests, being the clearest pattern of response to this dieback event. Overall, similar to other studies, the colihue dieback results in growth of advance regeneration, but it did not represent a major opportunity for new tree establishment in these forests.
\end{abstract}

Key words: regeneration dynamic, Saxegothaea conspicua, understory, gaps recruitment.

\section{RESUMEN}

En bosques templados lluviosos (> 600 m s.n.m.) de Sudamérica la bambúcea Chusquea culeou (colihue) es la especie dominante del sotobosque. Su floración y muerte puede generar una oportunidad para el establecimiento o crecimiento de la regeneración arbórea, especialmente en claros del dosel. En este estudio fue evaluado el efecto de esta alteración endógena sobre el establecimiento de especies arbóreas y el crecimiento de la regeneración avanzada en claros de dos bosques primarios -uno dominado por Nothofagus dombeyi-Saxegothaea conspicua-Dasyphyllum diacantoides y el otro dominado por Araucaria araucana-Nothofagus-, ubicados en la cordillera andina del centro-sur de Chile. En cada bosque (San Pablo de Tregua y Quetrupillán), entre los años 2001-2003 se registró el establecimiento del colihue post-floración y los cambios demográficos y el crecimiento de la regeneración arbórea en claros con colihue vivo y muerto. En ambos bosques, la mortalidad de la regeneración avanzada fue superior en claros con colihue muerto. Solamente en San Pablo de Tregua el crecimiento en altura de Saxegothaea conspicua fue mayor en claros con colihue muerto. El establecimiento de individuos post-floración fue bajo, sin presentar diferencias entre condiciones. El reclutamiento de plántulas de colihue fue superior en claros con colihue muerto en ambos bosques, siendo el patrón más claro de respuesta ante el evento de su propia muerte. La muerte del colihue puede favorecer el crecimiento de la regeneración avanzada pero sin representar una gran oportunidad para el establecimiento de especies arbóreas en estos bosques.

Palabras clave: dinámica regenerativa, Saxegothaea conspicua, sotobosque, regeneración en claros.

\section{INTRODUCCIÓN}

Numerosas especies de bambú presentan un patrón de floración, fructificación y muerte sincronizada en ciclos que pueden ser de varias décadas. Dentro del bosque el fenóme- no de floración de las bambúceas afecta el patrón de disponibilidad de recursos (luz, espacio, nutrientes, etc.) pudiendo generar un pulso de crecimiento rápido en la regeneración avanzada o el reclutamiento de nuevos individuos de especies arbóreas (Gratzer et al. 1999). Los efectos de este fe- 
nómeno sobre la regeneración arbórea, asociados con la heterogeneidad espacial y el incremento en la disponibilidad de recursos, pueden influenciar a largo plazo la diversidad y la estructura de las comunidades forestales donde participan estos bambúes (Beckage et al. 2000).

Bambúes del género Chusquea (Graminidae: Bambuseae) son característicos del sotobosque de los bosques templados del centro-sur $\left(37-43^{\circ}\right.$ S) de Chile (Matthei 1998). Las especies de Chusquea son especialmente abundantes en sitios perturbados (post-fuego, claros naturales o artificiales) y su habilidad para interferir en la dinámica regenerativa se encuentra bien documentada (Holz y Veblen 2006, Raffaele et al. 2007, Giordano et al. 2009, Muñoz y González 2009). Chusquea culeou (Desv.) (colihue) crece principalmente en claros de bosques ubicados en elevaciones medias y altas (Schlegel 1991), formando agrupaciones de 12 a 100 culmos que pueden llegar a medir más de $8 \mathrm{~m}$ de altura (Veblen et al. 1980, Veblen 1982). Según Veblen et al. (1980) el crecimiento de las plántulas de colihue luego de su muerte es lento, lo que podría generar una oportunidad para el establecimiento de especies arbóreas, especialmente para aquellas intolerantes a la sombra, las cuales son las especies pioneras en la sucesión de estos bosques. Estas observaciones y otros efectos ecológicos generados por este evento han sido estudiados aprovechando la ocurrencia de la última floración masiva del colihue (Raffaele et al. 2007, Giordano et al. 2009, Marchesini et al. 2009). La comprensión de este proceso ecológico es especialmente relevante en bosques antiguos, donde la dinámica de claros cumple un rol preponderante en la composición y estructura del bosque. Las floraciones de las especies de Chusquea han sido propuestas como las únicas alteraciones endógenas -producidas por agentes internos de la comunidad- potencialmente importantes para la regeneración de especies de Nothofagus, pioneras en la sucesión, en ausencia de alteraciones exógenas de gran escala (Veblen y Donoso 1987, Muñoz y González 2009).

A partir de la primavera del año 2000 el colihue floreció en un amplio rango latitudinal dentro de su distribución en Chile y Argentina ( $\left.\mathrm{ca} .39^{\circ}-42^{\circ} \mathrm{S}\right)$. Las principales poblaciones afectadas fueron aquellas asociadas a los bosques andinos dominados por Araucaria araucana (Mol.) y especies de Nothofagus (N. dombeyi Mirb., N. nervosa Phil., N. pumilio Poepp.). A diferencia de floraciones masivas ocurridas en otras especies (ej.: Chusquea quila (Kunth)), en que la mayor proporción de la población de plantas florecen y mueren (> 90 \%; González 2001), en colihue cierta proporción de la población queda sin florecer. Esta situación representa una excelente oportunidad para evaluar el efecto de su floración y muerte considerando parches de colihue sin florecer como control en el mismo bosque.

Ante este fenómeno, el objetivo central de este trabajo fue evaluar los cambios demográficos de la misma especie (colihue) y la respuesta en crecimiento y establecimiento de la regeneración arbórea durante las primeras dos estaciones de crecimiento post-muerte del colihue en dos bos- ques antiguos ubicados en la precordillera andina. Como hipótesis principal se plantea que la floración y muerte del colihue genera una oportunidad para el establecimiento de nuevos individuos arbóreos, principalmente de especies pioneras, como también genera un mayor crecimiento de la regeneración avanzada. De igual forma, el establecimiento del colihue se verá favorecido en claros donde la propia especie floreció y posteriormente murió.

\section{MÉTODOS}

Sitios de estudio. El estudio se llevó a cabo paralelamente en dos bosques antiguos entre los años 2001 y 2003. El primero de ellos se ubicó en el predio experimental San Pablo de Tregua ( $39^{\circ} 35^{`} \mathrm{~S}$ y $72^{\circ} 05^{`} \mathrm{O}$ ) y el otro en el sector Quetrupillán dentro del Parque Nacional Villarrica (39³ $38^{\prime} \mathrm{S}$ y $72^{\circ} 03^{\circ}$ O) (figura 1). En ambos sitios, el colihue era la especie dominante del sotobosque y a partir del año 2000 floreció, generando un mosaico de parches con colihue vivo (verde) y muerto (seco) bajo el bosque antiguo (figura 2). San Pablo de Tregua se encuentra a 900 m s.n.m., presenta una temperatura media anual del aire que varía entre 4 y $8{ }^{\circ} \mathrm{C}$, y una pluviosidad entre 3.000 y $4.000 \mathrm{~mm}$ anuales. Las especies arbóreas que componen su dosel dominante en el bosque de San Pablo de Tregua son: Nothofagus dombeyi, Laureliopsis philippiana (Looser), Saxegothaea conspicua (Lindl) y Dasyphyllum diacanthoides (Less.). El sector Quetrupillán se encuentra aproximadamente a 1.200 m s.n.m., según registros de la estación más próxima $(\mathrm{Pu}-$ cón: $230 \mathrm{~m}$ s.n.m.) la temperatura media anual del aire es de $9,7^{\circ} \mathrm{C}$ y la precipitación media anual alcanza $2.150 \mathrm{~mm}$ en el año. Las especies arbóreas dominantes en el bosque son: A. araucana, N. dombeyi, y marginalmente $N$. pumilio. Cada una de las áreas de estudio presenta una composición florística particular teniendo como elemento en común la presencia de colihue, que además es la especie del sotobosque de mayor cobertura y abundancia (cuadro 1).

Selección de claros e instalación de parcelas. En cada rodal de estudio se seleccionaron 10 claros de dosel, cinco presentaban colihue vivo sin florecer y cinco presentaban colihue florecido y muerto (seco). En cada claro se instaló una parcela permanente de $15 \times 15 \mathrm{~m}$ subdividida en 25 subparcelas de $3 \times 3 \mathrm{~m}$.

Respuesta de la regeneración arbórea. Se consideró regeneración arbórea a las plantas menores a $5 \mathrm{~cm}$ de diámetro a la altura del cuello de la planta. Para evaluar la respuesta de la regeneración avanzada (plantas de especies arbóreas establecidas antes de la muerte del colihue) y de los nuevos establecimientos (plantas establecidas después de la muerte del colihue), en diciembre de 2000 en cada claro se seleccionaron aleatoriamente cinco subparcelas de $3 \times 3 \mathrm{~m}$ donde se anidaron parcelas de $1 \times 1 \mathrm{~m}$. Estas últimas parcelas fueron monitoreadas durante los años 2001, 2002 y 2003. En cada una se registró el establecimiento de nuevos indi- 


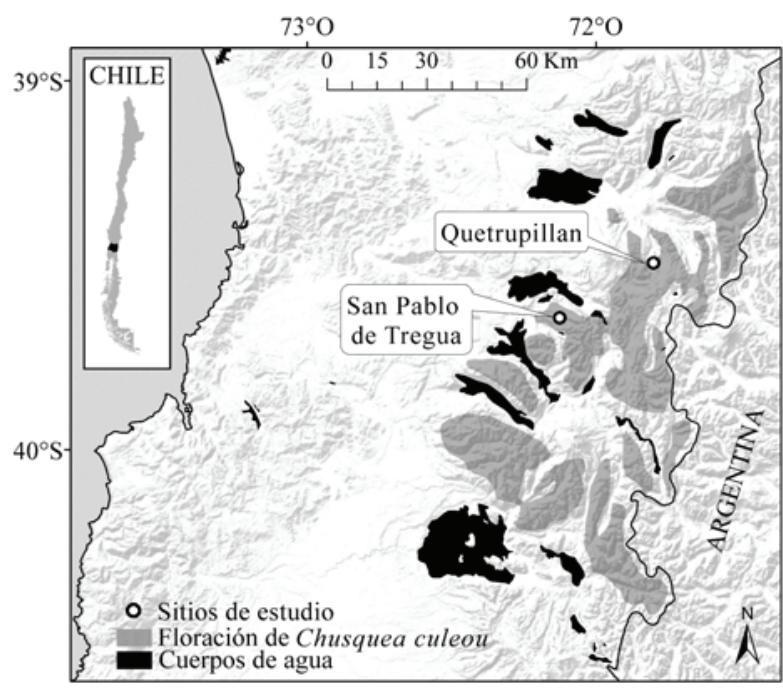

Figura 1. Ubicación geográfica de los bosques estudiados (San Pablo de Tregua, Quetrupillán) y distribución aproximada de la floración de Chusquea culeou (colihue) en Chile entre los años 2000-2002.

Geographic location of the studied forests (San Pablo de Tregua, Quetrupillán). The approximate distribution of Chusquea culeou (colihue) between 2000 and 2002 is indicated in white stripes. viduos y la mortalidad y crecimiento en altura de la regeneración avanzada. De esta forma, en cada condición (claros con colihue vivo, claros con colihue muerto) se monitoreó una superficie agregada de $25 \mathrm{~m}^{2}$. En el caso de la regeneración avanzada de $N$. dombeyi también se registró el sustrato en el cual se encontró creciendo (hojarasca o sobre-tronco).

Respuesta de la regeneración de colihue. En cada parcela de 1 x $1 \mathrm{~m}$ utilizada para evaluar la respuesta de la regeneración arbórea se anidó una subparcela de $0,3 \times 0,3 \mathrm{~m}$ donde se monitoreó la densidad de plántulas de colihue. Estas plántulas también fueron censadas durante los años 2001, 2002 y 2003.

Cobertura de las especies del sotobosque. Dentro de las 25 subparcelas de $3 \times 3$ m establecidas en cada uno de los claros, durante el año 2001 se seleccionaron aleatoriamente cinco subparcelas para estimar el porcentaje de cobertura según Braun-Blanket (Van der Maarel 1979). De esta forma el monitoreo de la cobertura del sotobosque representó una superficie agregada de $45 \mathrm{~m}^{2}$ en claros con colihue vivo, y la misma superficie en claros con colihue muerto. El valor medio de cada rango de cobertura fue utilizado para el cálculo del valor de importancia de las especies de sotobosque.

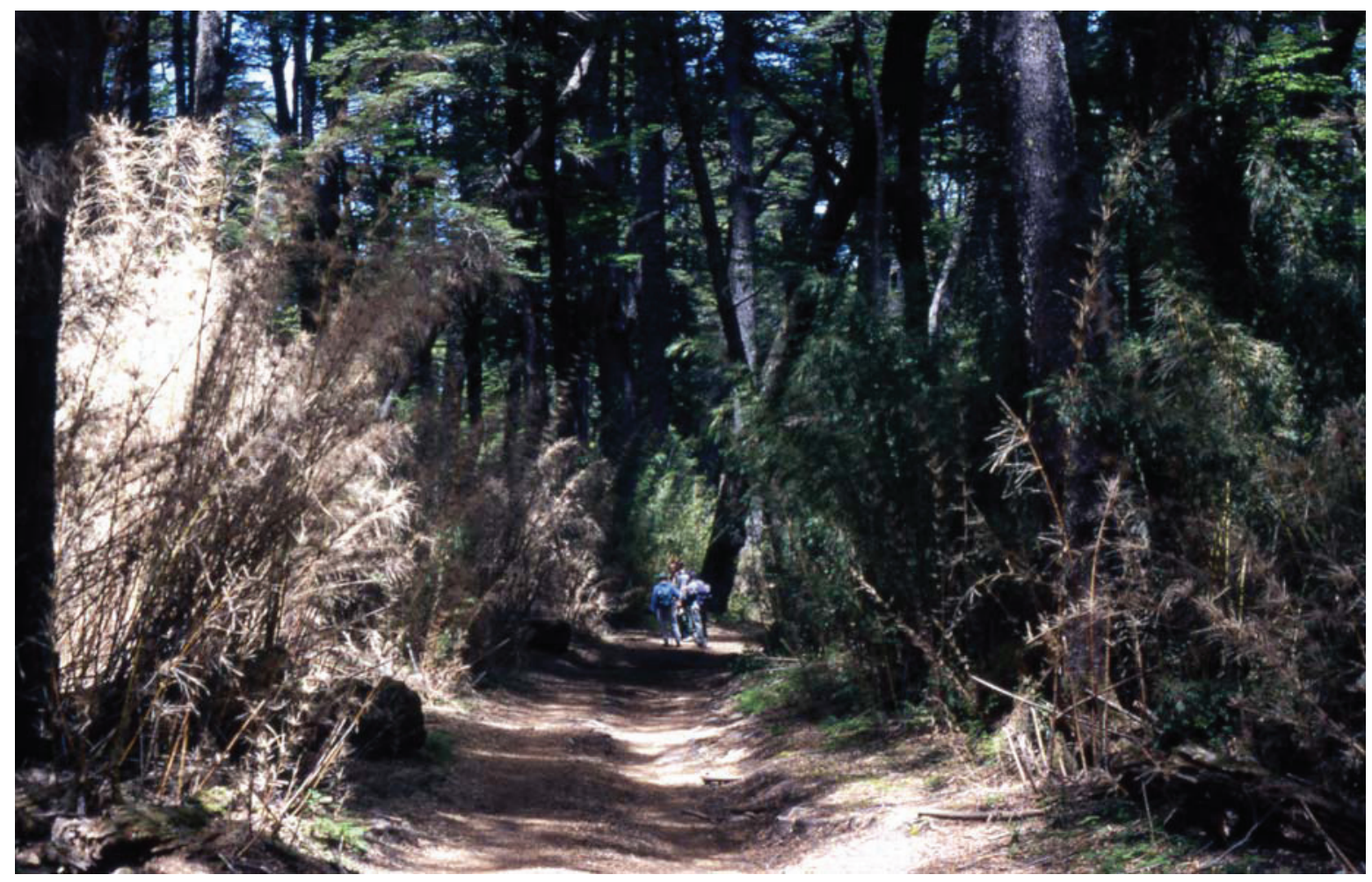

Figura 2. Parches de Chusquea culeou (colihue) vivo y muerto dentro del bosque. Los parches con colihue muerto y vivo se observan mayoritariamente a la izquierda y derecha del camino, respectivamente. Fotografía M.E. González.

Live and dead patches of $C$. culeou (colihue) within forest gaps. Colihue dead and alive patches are seen mostly on the left and right of the road, respectively. 
Cuadro 1. Valor de importancia de las especies presentes en los claros con Chusquea culeou (colihue) vivo y muerto en los bosques de Quetrupillán y San Pablo de Tregua.

Importance value of species in gaps dominated by live and dead C. culeou in Quetrupillán and San Pablo de Tregua forests.

\begin{tabular}{|c|c|c|c|c|}
\hline \multirow{3}{*}{ Especies del sotobosque } & \multicolumn{4}{|c|}{ Valor de importancia (\%) } \\
\hline & \multicolumn{2}{|c|}{ Quetrupillán } & \multicolumn{2}{|c|}{ San Pablo de Tregua } \\
\hline & Muerto & Vivo & Muerto & Vivo \\
\hline \multicolumn{5}{|l|}{ Arbustos } \\
\hline Chusquea culeou (Desv.) & 111,3 & 71,4 & 67 & 75,3 \\
\hline Drimys andina (Reiche) R.A.Rodr. et Quez. & 20,4 & 21,4 & - & - \\
\hline Desfontainea spinosa (Ruiz et Pavón) & 11 & 42 & - & - \\
\hline Maytenus disticha (Hook.f) Urban. & 11 & 11,3 & - & - \\
\hline Myoschilos oblonga (Ruiz et Pavón) & 6,2 & 2 & - & - \\
\hline Myrceugenia chrysocarpa (Berg) Kausel & 6,3 & 5,1 & - & - \\
\hline Gaultheria mucronata (L. f.) Hook. et Arn. & 28 & 47 & - & - \\
\hline Maytenus magellanica (Lam.) Hook. f. & 4 & - & - & - \\
\hline Berberis sp. & 1,4 & - & - & - \\
\hline Azara lanceolata (Hook. f.) & - & - & 9,1 & 10 \\
\hline Myrceugenia planipes (Hook. et Arn.) Berg. & - & - & 2,1 & 4 \\
\hline \multicolumn{5}{|l|}{ Trepadoras y rastreras } \\
\hline Luzuriaga radicans (Ruiz et Pavón) & - & - & 7 & 6 \\
\hline Mitraria coccinea (Cav.) & - & - & 39 & 4 \\
\hline Griselinia ruscifolia (Clos) Ball. & - & - & 4 & 1,3 \\
\hline Campsidium valdivianum (Phil.) Skottsb. & - & - & 8 & 30,4 \\
\hline Hydrangea serratifolia (H. et A.) Phil. f. & - & - & 16 & 1,3 \\
\hline Nertera granadensis (Mutis ex 1.f.) Druce. & - & - & 1 & 3 \\
\hline \multicolumn{5}{|l|}{ Herbáceas } \\
\hline Alstroemeria sp. & 1 & - & - & - \\
\hline Dysopsis glechomoides (A. Rich.) Muell. Arg. & - & - & 7 & 14,4 \\
\hline Loasa acanthifolia (Desr.) & - & - & 16 & 19 \\
\hline Urtica dioica (L.) & - & - & 2 & - \\
\hline \multicolumn{5}{|l|}{ Helechos y epifitas } \\
\hline Blechnum blechnoides (Keyserl.) & - & - & 12 & 14 \\
\hline Ctenitis spectabilis (Kaulf.) Kunkel & - & - & 3 & 1,1 \\
\hline Greigia landbeckii (Lechl. ex Phil.) Phil. & - & - & 4,1 & 9 \\
\hline \multicolumn{5}{|l|}{ Musgos } \\
\hline Dendroligotrichum dendroides (Hedw.) Broth. & - & - & 6 & 11 \\
\hline
\end{tabular}

Análisis de datos. El valor de importancia de las especies del sotobosque de los claros se obtuvo a partir de la suma de la cobertura relativa más la frecuencia relativa que obtuvo cada especie en las parcelas de $3 \times 3 \mathrm{~m}$. La cobertura relativa se calculó utilizando el valor medio del rango de cobertura según Braun-Blanquet (Van der Maarel 1979). Las diferencias en densidad y en incremento en altura de las plantas entre claros con colihue seco y claros con colihue verde se evaluaron utilizando la prueba $U$ de MannWhitney a un $95 \%$ de confianza.

\section{RESULTADOS}

Respuesta de la regeneración avanzada de especies arbóreas. En Quetrupillán la regeneración avanzada estaba compuesta (año 2001) por N. dombeyi, A. araucana y $N$. pumilio. Luego de dos temporadas de crecimiento post-floración (2001-2002, 2002-2003) la mortalidad en las tres especies fue mayor en claros con colihue muerto (seco) en relación a los claros con colihue vivo (figura 3). 


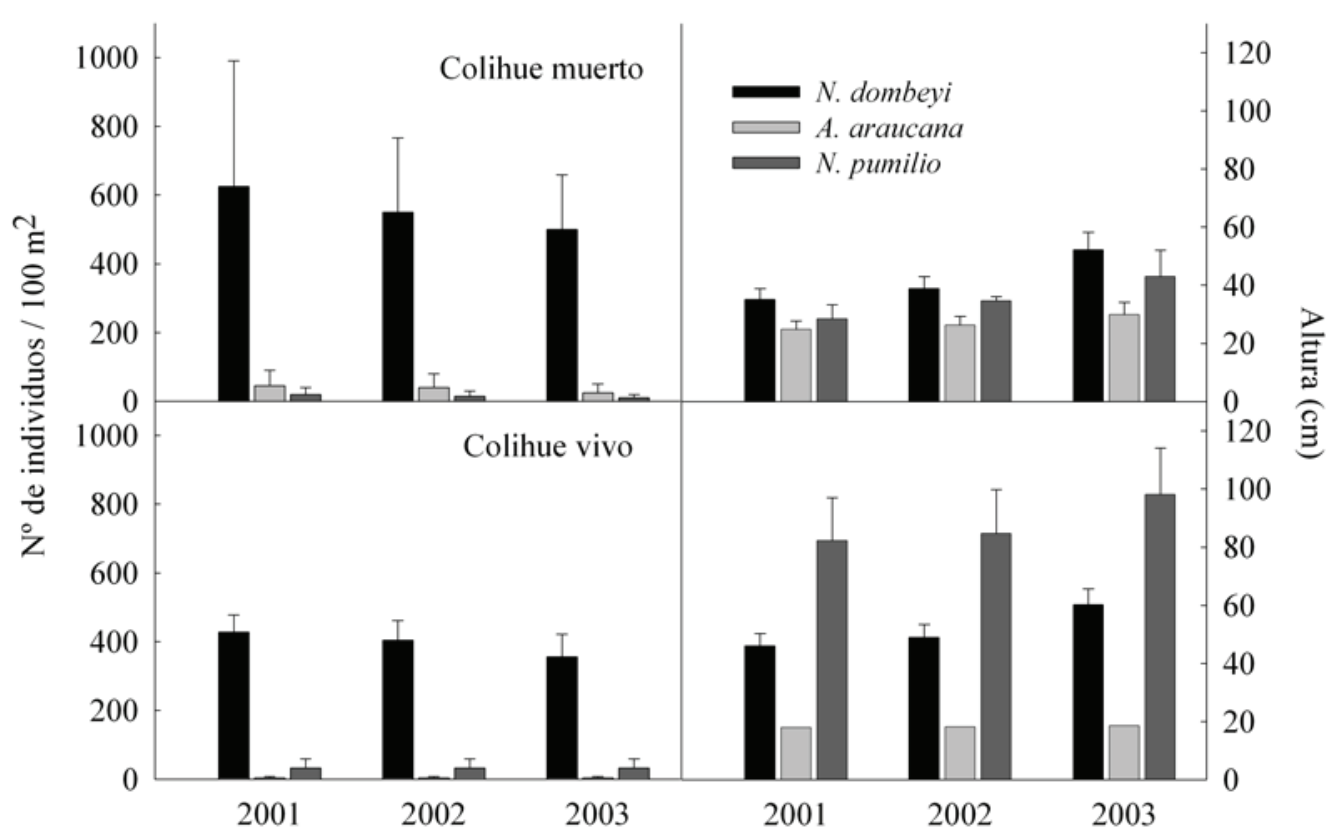

Figura 3. Cambios demográficos (izquierda) y crecimiento promedio (derecha) en altura de la regeneración avanzada de especies arbóreas durante las tres evaluaciones (años 2001, 2002, 2003) en claros con Chusquea culeou (colihue) vivo (inferior) y muerto (superior) en el sector Quetrupillán. Las barras de error corresponden al error estándar de la media.

Demographic changes (left) and mean growth (right) in height of advance tree regeneration for the three evaluated periods (years 2001, 2002, 2003) in gaps with live and dead C. culeou in Quetrupillán. The error bars correspond to standard error.

Nothofagus dombeyi presentó una mortalidad de $34 \%$ al cabo del periodo evaluado (2001-2003) en claros con colihue muerto, mientras en claros con colihue vivo (verde) su mortalidad solo alcanzó $17 \%$ en el mismo periodo. En este bosque $N$. dombeyi mostró mayor abundancia sobre un sustrato de hojarasca (figura 4A). Por su parte A. araucana evidenció una mortalidad de $44 \%$ en claros con colihue muerto. Fueron muy pocos los individuos de esta especie en claros con colihue vivo para observar un patrón claro de mortalidad. Nothofagus pumilio mostró una mortalidad de $50 \%$ en claros con colihue muerto mientras en claros con colihue vivo no presentó mortalidad de individuos.

El crecimiento en altura de las tres especies en Quetrupillan fue similar (figura 3) y no presentó diferencias significativas entre claros con colihue vivo y muerto (MannWitney; $P<0,05)$.

La regeneración avanzada en el bosque de San Pablo de Tregua se encontraba compuesta (año 2001) por L. philippiana, S. conspicua, D. diacantoides y $N$. dombeyi. Esta última especie se observó creciendo mayoritariamente sobre un sustrato de troncos caídos en el piso del bosque (figura 4B). Únicamente $L$. philippiana presentó mayor mortalidad en claros con colihue muerto (12\%) respecto de claros con colihue vivo (3\%) durante el periodo total evaluado. Las demás especies mostraron valores similares de mortalidad en ambas situaciones (figura 5).

La regeneración de $S$. conspicua presentó un crecimiento significativamente superior en claros con colihue

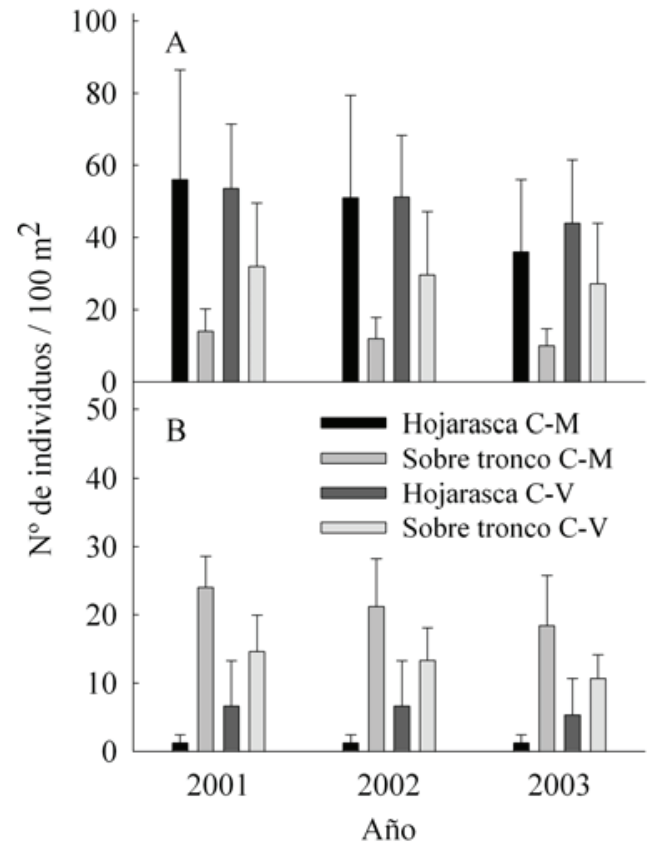

Figura 4. Proporción de individuos de Nothofagus dombeyi (regeneración avanzada) creciendo sobre un sustrato de hojarasca o sobre troncos caídos en claros con Chusquea culeou (colihue) muerto (C-M) y vivo (C-V) en el bosque de Quetrupillán (A) y San Pablo de Tregua (B).

Abundance of $N$. dombeyi advance regeneration established on litter and on fallen logs in gaps with live (C-V) and dead (C-M) $C$. culeou in Quetrupillán (A) and in San Pablo de Tregua (B). 


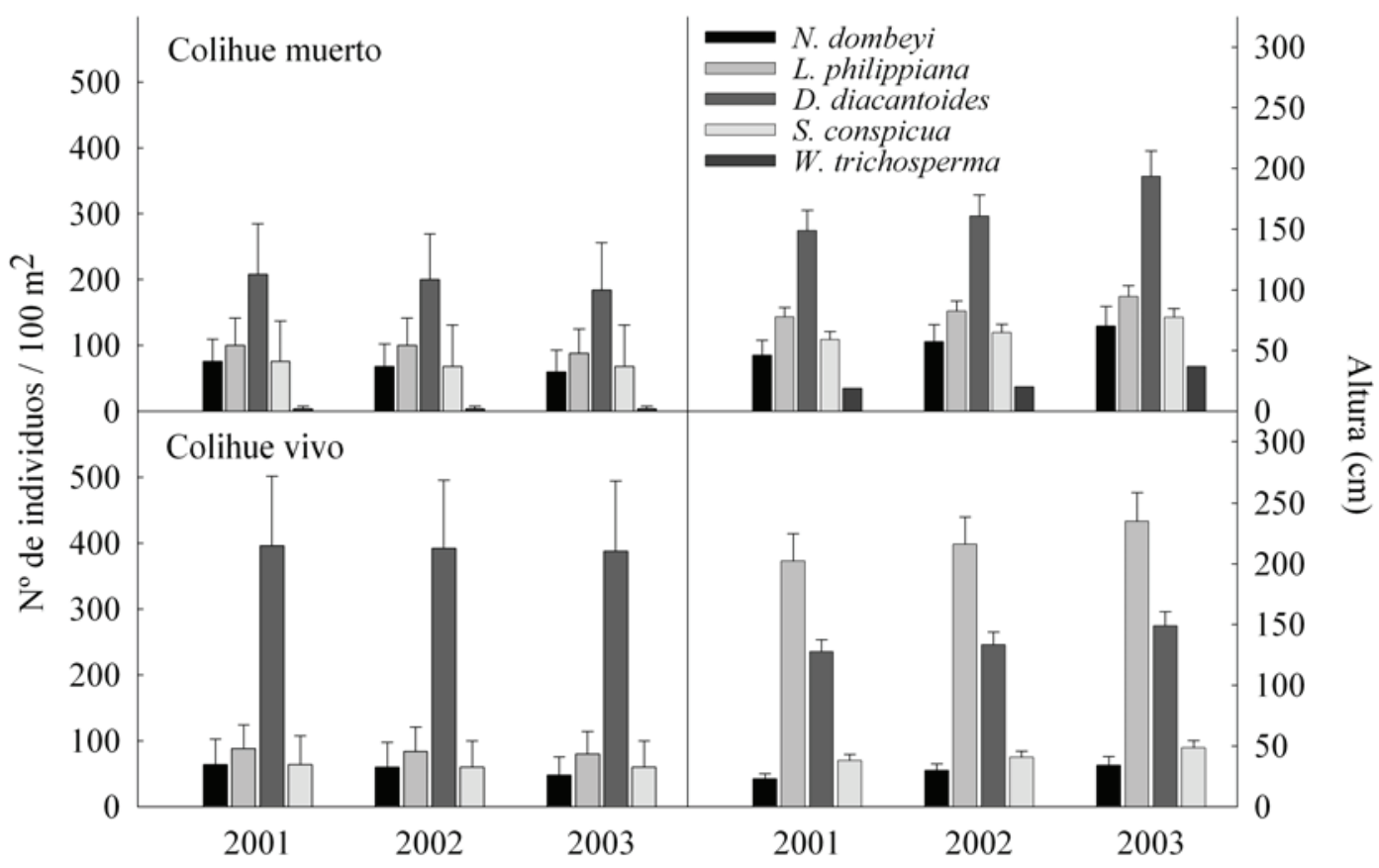

Figura 5. Cambios demográficos (izquierda) y crecimiento promedio (derecha) en altura de la regeneración avanzada de especies arbóreas durante las tres evaluaciones (años 2001, 2002, 2003) en claros con Chusquea culeou (colihue) vivo (inferior) y muerto (superior) en el bosque de San Pablo de Tregua. Las barras de error corresponden al error estándar de la media.

Demographic changes (left) and mean growth (right) in height of advance tree regeneration for the three evaluated periods (years 2001, 2002, 2003) in gaps with live and dead C. culeou in San Pablo de Tregua. Error bars correspond to standard error.

muerto respecto de claros con colihue vivo (Mann-Witney; $P<0,05)$. Estas diferencias ocurrieron tanto en la segunda estación de crecimiento 2002-2003 ( $U$ = -2,505; $P=0,012)$ como durante el periodo total evaluado 20012003 ( $U=-2,494 ; P=0,013$ ) (figura 5). El crecimiento medio de la regeneración de $D$. diacantoides y $N$. dombeyi no presentó diferencias significativas entre condiciones. Por su parte, L. philippiana presentó mayores incrementos en altura en claros con colihue vivo respecto de claros con colihue muerto durante la primera estación de crecimiento post-floración 2001-2002 $(U=-2,750 ; P=0,006)$ y el periodo total evaluado (2001-2003) ( $U=-2,156$; $P=0,031)$.

Regeneración arbórea post-floración. En Quetrupillán el establecimiento de la regeneración de $N$. dombeyi y A. araucana post-floración (nuevos establecimientos) no presentó diferencias significativas entre claros con colihue vivo y muerto (Mann-Whitney; $P<0,05$ ) (figura 6A).

En el bosque de San Pablo de Tregua los nuevos establecimientos fueron principalmente de las especies L. philippiana y S. conspicua. En ninguna de las especies el establecimiento presentó diferencias significativas entre claros con colihue vivo y muerto (Mann-Whitney; $P<0,05$ ). En la tercera evaluación (2003) L. philippiana no presentó sobrevivientes en claros con colihue muerto (figura 6B).
Establecimiento de colihue post-floración. El establecimiento de plántulas de colihue en claros donde esta especie había muerto en Quetrupillán, fue significativamente superior durante las tres evaluaciones (2001 $U=-4,142$, $P=0,000 ; 2002 U=-3,658, P=0,000 ; 2003 U=-5,406$, $P=0,000)$. El establecimiento de esta especie en dicha condición superó en tres, diez y cinco veces su establecimiento en claros con colihue vivo en las evaluaciones del 2001, 2002 y 2003, respectivamente (figura 7).

En el bosque de San Pablo de Tregua, el establecimiento del colihue durante las primeras dos evaluaciones postfloración fue superior en claros con colihue muerto (2001: $U=-4,207, P=0,000 ; 2002: U=-1,997, P=0,046)$. Aunque en la tercera evaluación el patrón de establecimiento fue el mismo (mayor en claros con colihue muerto), estas diferencias no alcanzaron niveles significativos (figura 7). Durante el periodo total evaluado (2001-2003) la mortalidad de la regeneración de esta especie fue $40 \%$ superior en claros con colihue vivo respecto de claros con colihue muerto (figura 7).

\section{DISCUSIÓN}

Efecto de la muerte del colihue sobre la regeneración avanzada. En el bosque de San Pablo de Tregua fue abundante la regeneración avanzada en claros, tanto de especies 


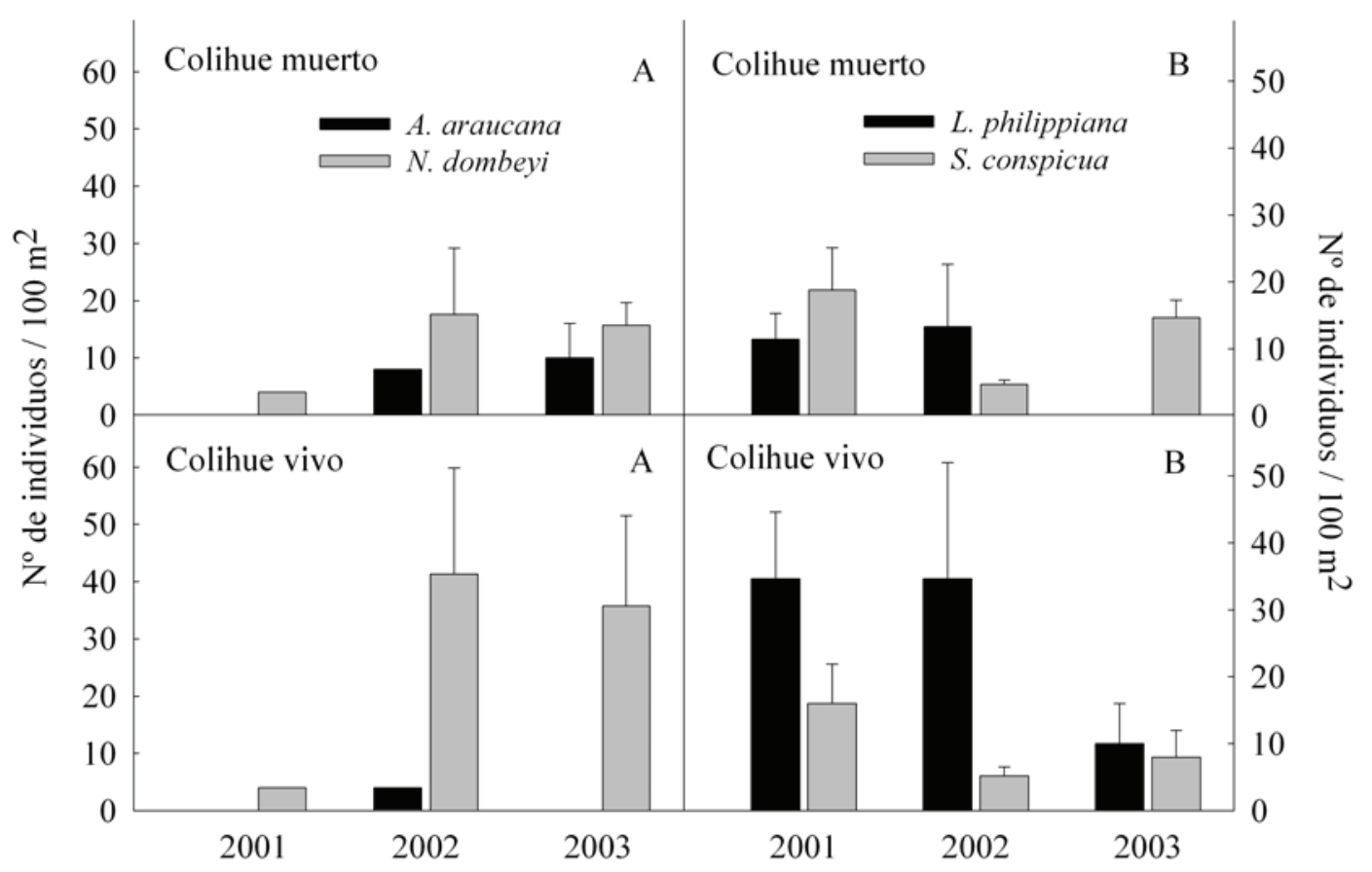

Figura 6. Cambios demográficos de los nuevos establecimientos arbóreos durante las tres evaluaciones (2001, 2002, 2003) en el bosque de Quetrupillán (A) y San Pablo de Tregua (B) en claros con Chusquea culeou (colihue) vivo y muerto.

Demographic changes of the new tree regeneration for the three evaluated periods (years 2001, 2002, 2003) in gaps with live and dead $C$. culeou in Quetrupillán (A) and San Pablo de Tregua (B) forest.

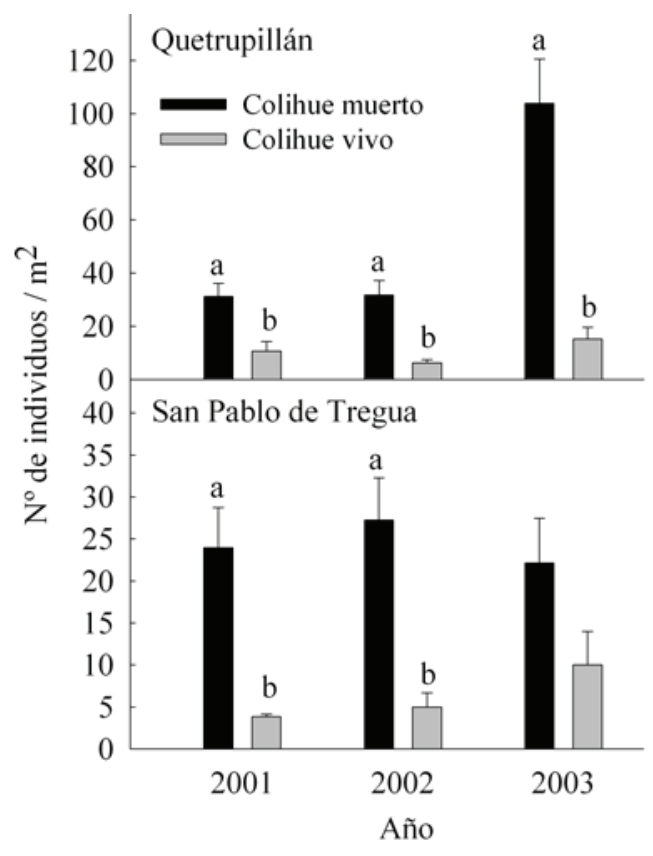

Figura 7. Densidad de plántulas de Chusquea culeou (colihue) en los bosques estudiados en claros con colihue vivo y muerto. Letras diferentes sobre las barras indican diferencias significativas entre condiciones (colihue vivo-muerto) en cada año de evaluación.

Seedling density of $C$. culeou for the three evaluated periods (years 2001, 2002, 2003) in gaps with live and dead C. culeou in Quetrupillán and San Pablo de Tregua forest. tolerantes como de individuos de $N$. dombeyi establecidos principalmente sobre troncos caídos. En el caso del bosque de Quetrupillán, se encontró también una abundante regeneración de $N$. dombeyi en claros, pero establecida mayormente sobre el sustrato de hojarasca. La distinta estructura y composición de los bosques en Quetrupillán y San Pablo de Tregua pueden explicar las diferencias en la abundancia de individuos de $N$. dombeyi y el tipo de sustrato para la regeneración de esta especie. Una estructura del bosque más abierta en Quetrupillán, sin la participación de especies tolerantes en los doseles codominantes, intermedios e inferiores, permitió el establecimiento más abundante de $N$. dombeyi en el piso del bosque. Al contrario, la mayor densidad y cobertura de los bosques en San Pablo de Tregua -incluyendo colihuales más densos y altos-, resultó en un establecimiento menos abundante y preferentemente sobre troncos caídos. En los dos bosques analizados un mayor número de troncos caídos en el suelo de los claros podría explicar en gran parte las diferencias en la abundancia de la regeneración avanzada de $N$. dombeyi entre condiciones (colihue vivo y muerto). En este sentido se encuentra bien documentado que la regeneración de los coihues $(N$. dombeyi, Nothofagus betuloides (Mirb.) y Nothofagus nitida (Phil.)) en claros de bosques antiguos requiere de aberturas de gran tamaño en el dosel (Veblen et al. 1981, Pollmann y Veblen 2004), y en muchos casos también de troncos en el suelo (Lusk 1995, Christie et al. 2003). 
En general, la floración y muerte del colihue pareció afectar negativamente la sobrevivencia de la regeneración avanzada de especies arbóreas en los claros. Este tipo de regeneración presentó mayor mortalidad en la condición de colihue muerto, especialmente en el sitio de Quetrupillán, donde todas las especies arbóreas disminuyeron su abundancia. Este patrón de mortalidad ha sido previamente documentado asociado principalmente al shock (o desequilibrio) que sufren las plantas -especies tolerantes a la sombra creciendo en el sotobosque- luego del cambio de las condiciones ambientales producto del desmoronamiento de la cobertura de bambúceas del género Chusquea (González et al. 2002, Campanello et al. 2007, Muñoz y González 2009).

Dependiendo del sitio de estudio, el crecimiento en altura de la regeneración avanzada fue favorecido o no influenciado por la floración y muerte del colihue. Mientras en los claros del bosque de Quetrupillán el crecimiento fue similar en ambas condiciones (colihue vivo y muerto), en el bosque de San Pablo de Tregua la regeneración avanzada, especialmente de $S$. conspicua, presentó un mayor crecimiento en altura como respuesta a la muerte y consiguiente pérdida de la cobertura del bambú en los claros. La liberación de individuos arbóreos en claros asociado a la muerte o remoción de especies de Chusquea ha sido también documentada por diferentes autores (González et al. 2002, Holz y Veblen 2006, Campanello et al. 2007, Marchesini et al. 2009, Muñoz y González 2009).

Efecto de la muerte del colihue sobre la regeneración arbórea post-floración. Este disturbio endogénico no constituyó un factor clave en el establecimiento de especies arbóreas en los claros. Tanto en San Pablo de Tregua como en Quetrupillán, la especie pionera en ambos bosques ( $N$. dombeyi) no parece depender de la ventana temporal en la que los claros quedaron desprovistos de la cobertura del bambú. De hecho, en Quetrupillán el establecimiento de $N$. dombeyi fue mayor en claros con colihue vivo. En otros tipos de bosques, estos eventos tampoco han ofrecido una oportunidad para el establecimiento de especies de Nothofagus. Por ejemplo, después de la muerte de C. quila, Nothofagus obliqua (Mirb.) presentó un abundante establecimiento inicial, sin embargo, sus bajos crecimientos respecto de la regeneración del bambú, como probablemente también el pequeño tamaño de los claros analizados, determinaron la nula sobrevivencia de los individuos de esta especie al cabo de 10 años post-floración (Muñoz y González 2009). Tampoco fue superior el establecimiento de $N$. dombeyi y N. pumilio en parches donde floreció Chusquea montana (Phil.) en zonas altas de Los Andes (Holz y Veblen 2006). Similar situación fue documentada para $N$. nervosa, que no presentó regeneración post-floración de colihue, en bosques ubicados en la vertiente oriental de Los Andes (Marchesini et al. 2009), a pesar que esta especie es más tolerante a la sombra que $N$. dombeyi y $N$. obliqua en etapas iniciales de establecimiento (Mueller-Using y Schlegel 1981).
Como ha sido observado, la floración y muerte de las especies de Chusquea genera micrositios que pueden favorecer la germinación y establecimiento inicial de especies de Nothofagus (González et al. 2002, Giordano et al. 2009). Estos últimos procesos son principalmente favorecidos por la remoción del horizonte orgánico - con gran acumulación de hojarasca seca de bambú - por aves del sotobosque. Sin embargo, las etapas más críticas para el definitivo establecimiento de la regeneración de estas especies parecen producirse con la competencia de la nueva cohorte de Chusquea spp. (Muñoz y González 2009).

Para el caso de la especie semi-tolerante A. araucana, este disturbio tampoco resultó en un factor determinante que favoreciera el reclutamiento de la especie. Si bien la regeneración de Araucaria araucana es favorecida en sitios abiertos, alejados de la cobertura de colihue (Finckh y Paulsch 1995), la biología y el ciclo reproductivo de la especie - dioica y de una producción bianual de semillas irregular - y sus mecanismos de diseminación juegan un papel más preponderante en su capacidad y éxito de establecimiento (Veblen 1982, González et al. 2006).

Por su parte, en el bosque de San Pablo de Tregua la muerte de colihue tampoco favoreció el establecimiento de especies arbóreas, como queda demostrado por el exiguo reclutamiento de especies de mayor tolerancia a la sombra, que suelen dominar el bosque en estados sucesionales tardíos. Similar respuesta ha sido encontrada por Muñoz y González (2009) y Campanello et al. (2007), luego de la pérdida de la cobertura de C. quila y Chusquea ramosissi$m a$ (Lindm.), respectivamente. Un aumento de la regeneración de $S$. conspicua en claros con colihue muerto fue observado, aunque sin alcanzar niveles significativos esta especie logró duplicar su participación en densidad respecto de claros con colihue vivo al final del periodo evaluado (2001-2003). Posiblemente la capacidad de esta especie para mantener un banco de semillas, le brinde una ventaja competitiva para responder a este evento en comparación con otras especies (ej.: Nothofagus spp.) que no forman un banco de semillas persistente (Cuevas y Arroyo 1999), las cuales dependerían de la coincidencia de este evento con años de alta producción de semillas para aumentar su regeneración durante los primeros años post-floración (González et al. 2002).

Patrón de regeneración de colihue post-muerte de la generación anterior. El mayor establecimiento de plántulas de C. culeou en los claros donde esta especie murió, en ambos bosques, sugiere una alta efectividad en su mecanismo de autofacilitación de su regeneración. Esta estrategia de regeneración ha sido un patrón común en los recientes estudios acerca de las repercusiones de las floraciones de las especies del género Chusquea de los bosques templados de Chile y Argentina (González et al. 2002, Holz y Veblen 2006, Raffaele et al. 2007, Marchesini et al. 2009, Muñoz y González 2009). Diferente fue la respuesta del sotobosque en los bosques atlánticos de Misiones donde 
C. ramosissima fue remplazada por un sotobosque más abierto compuesto por especies de herbáceas, helechos y piperáceas (Campanello et al. 2007, Gallardo et al. 2008).

Considerando la escasa o relativamente lenta respuesta inicial de la regeneración arbórea después de la muerte de colihue, esta especie, al igual que C. quila (Muñoz y González 2009), mantendrá su dominancia en los claros por varias décadas hasta que sucesores potenciales de especies principalmente tolerantes logren alcanzar y posicionarse en el dosel inhibiendo la vigorosidad y cobertura de colihue.

Junto con la rápida respuesta de las especies de Chusquea spp. en distintos bosques, el mayor crecimiento en altura (liberación) de la regeneración avanzada ha sido el patrón más recurrente como respuesta a la floración y muerte de estos bambúes. Por su parte, el rol que juega el evento de muerte de especies de Chusquea spp. sobre el reclutamiento de especies arbóreas y la dinámica regenerativa dentro del bosque, podría ser dependiente de una combinación más compleja de factores, como pueden ser características del claro (edad, origen, tamaño, forma), abundancia de fuentes de semilla y de legados biológicos (troncos, tocones, árboles muertos en pie) y el ensamble de especies arbóreas y sus habilidades competitivas en los bosques donde ocurre el evento (González et al 2002, Holz y Veblen 2004, Muñoz y González 2009).

\section{CONCLUSIONES}

En relación a la hipótesis que la floración y muerte de colihue favorece el establecimiento de las especies arbóreas principales, para ambos tipos de bosques -San Pablo de Tregua y Quetrupillán-, el establecimiento de individuos arbóreos no fue influenciado por este proceso ecológico. Tanto las especies pioneras (ej., $N$. dombeyi, A. araucana) como aquellas de sucesión tardía (S. conspicua, L. philippiana) no parecen depender ni ser favorecidas por el evento de floración. Sin embargo, la floración y muerte del colihue afecta negativamente la sobrevivencia de la regeneración avanzada en los claros, la cual presenta mayor mortalidad en la condición de colihue muerto. En términos del crecimiento en altura de la regeneración avanzada -bajo condiciones de colihue vivo y muerto-, sólo S. conspicua, en el bosque de San Pablo de Tregua, presenta mayor crecimiento en altura como respuesta a la muerte y consiguiente pérdida de la cobertura del bambú en los claros. El patrón más evidente de respuesta ante el evento de muerte del colihue fue el de la propia especie, la cual presentó una rápida colonización vía semillas de la generación anterior en los claros donde perdió su cobertura como consecuencia de la muerte y posterior desmoronamiento de su biomasa.

\section{AGRADECIMIENTOS}

Los autores agradecen a National Geographic Science y a la Corporación Nacional Forestal (CONAF) de la región de La Araucanía. También a Ignacio Díaz, Andrés
Holz, Carlos Le Quesne, Jonathan Barichivich, Jason Sibold, Eduardo Aguallo y Hugo Álvarez.

\section{REFERENCIAS}

Baskin J. 2009. Death of Bamboo triggers regeneration of overstory tree in a southern beech forest. New Phytologist 181: 749-751.

Beckage B, JS Clark, BD Clinton, BL Haines. 2000. A long-term study of tree seedling recruitment in Southern Appalachian forests: the effects of canopy gaps y shrub understories. Canadian Journal of Forest Research 30: 1617-1631.

Campanello P, M Gatti, A Ares, L Montii, G Goldstein. 2007. Tree regeneration and microclimate in a liana and bamboodominated semideciduous Atlantic Forest. Forest Ecology and Management 252: 108-117.

Christie D, J Armesto. 2003. Regeneration microsites and tree species coexistence in temperate rain forests of Chiloé Island, Chile. Journal of Ecology 91(5): 776-784.

Cuevas J, M Arroyo. 1999. Ausencia de banco de semillas persistente en Nothofagus pumilio en Tierra del Fuego, Chile. Revista Chilena de Historia Natural 72:73-82.

Finckh M, A Paulsch. 1995. The ecological strategy of Araucaria araucana. Flora 190(4): 365-382.

Gallardo A, L Montti, S Bravo. 2008. Efectos del tacuarembó (Chusquea ramosissima) sobre el proceso de dispersión de semillas en la Selva Misionera. Ecología Austral 18: 347-356.

Giordano C, R Sánchez, A Austin. 2009. Gregarious bamboo flowering opens a window of opportunity for regeneration in a temperate forest of Patagonia. New Phytologist 181: 880-889.

González ME. 2001. Fenología de Chusquea quila durante su floración gregaria en el centro-sur de Chile. Bosque 22(2): 45-51.

González ME, TT Veblen, C Donoso, L Valeria. 2002. Tree regeneration responses in a lowland Nothofagus-dominated forest after bamboo die-back in south-central Chile. Plant Ecology 161: 59-73.

González ME, M Cortes, F Izquierdo, L Gallo, C Echeverría, S Bekessy, P Montaldo. 2006. Coníferas chilenas: Araucaria araucana. In Donoso C ed. Las especies arbóreas de los bosques Templados de Chile y Argentina. Autoecología. Valdivia, Chile. Marisa Cuneo Ediciones. p. 36-53.

González ME, TT Veblen, J Sibold. 2010. Influence of fire severity on stand development of Araucaria araucana - Nothofagus pumilio stands in the Andean cordillera of south-central Chile. Austral Ecology 35: 597-615.

Gratzer G, PB Rai, G Glatzel. 1999. The influence of the bamboo Yushania microphila on regeneration of Abies densa in central Bhutan. Canadian Journal of Forest Research 29: 1518-1527.

Holz CA, TT Veblen. 2006. Tree regeneration responses to Chusquea montana bamboo die-off in a subalpine Nothofagus forest in the southern Andes. Journal of Vegetation Science 17: 19-28.

Lusk C. 1995. Seed Size, Establishment Sites and Species Coexistence in a Chilean Rain Forest. Journal of Vegetation Science 6: 249-256

Marchesini V, O Sala, A Austin. 2009. Ecological consequences of massive flowering event of bamboo (Chusquea culeou) 
in temperate forest of Patagonia, Argentina. Journal of Vegetation Science 20: 424-432.

Matthei O. 1998. Las especies del genero Chusquea Kunth (Poaceae: Bambusoideae), que crecen en la X Región, Chile. Gayana Botánica 54 (2): 199-220.

Mueller-Using B, F Schlegel. 1981. The development of seedlings of Chilean Nothofagus species in a shaded area. Plant Research Development 13: 152-184.

Muñoz AA, ME González. 2009. Patrones de regeneración arbórea en claros a una década de la floración y muerte masiva de Chusquea quila (Poaceae) en un remanente de bosque antiguo del valle central en el centro-sur de Chile. Revista Chilena de Historia Natural 82: 185-198.

Pollmann W, T Veblen. 2004. Nothofagus regeneration dynamics in south-central Chile: a test of a general model. Ecological Monographs 74 (4): 615-634.

Raffaele E, T Kitzberger, T Veblen. 2007. Interactive effects of introduced herbivores and post-flowering die-off of bamboos in Patagonian Nothofagus forests. Journal of Vegeta- tion Science 18(3): 371-378.

Schlegel F. 1991. Growth behavior of Chusquea culeou Desv. in South-Central Chile. American Bamboo Society 8: 59-65.

Van der Maarel E. 1979. Transformation of cover-abundance values in phytosociology and its effects on community similarity. Vegetatio 39(2): 97-114.

Veblen TT, FM Schlegel, R Escobar. 1980. Structure and dynamics of old-growth Nothofagus forests in the Valdivian Andes, Chile. Journal of Ecology 68: 1-31.

Veblen TT, C Donoso, FM Schlegel, R Escobar. 1981. Forest dynamics in south-central Chile. Journal of Biogeography 8: 211-247.

Veblen TT. 1982. Growth patterns of Chusquea bamboos in the understory of Chilean Nothofagus forests and their influences in forest dynamics. Bulletin of the Torrey Botanical Club 109: 474-487.

Veblen T, C Donoso. 1987. Alteración natural y dinámica regenerativa de las especies chilenas de Nothofagus de la región de Los Lagos. Bosque 8(2): 133-142.

Recibido: 04.03.11

Aceptado: 16.04.12 